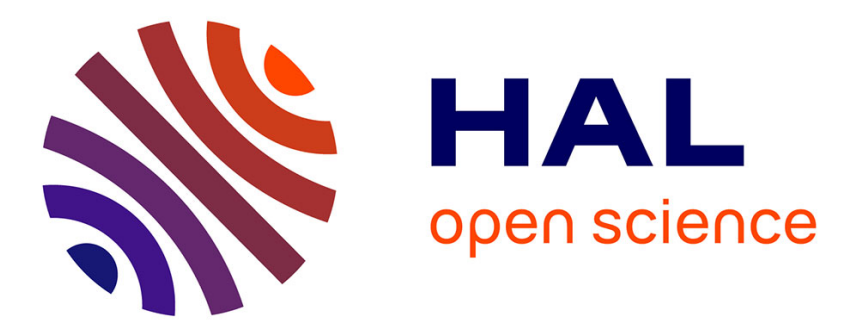

\title{
Relaxation rate distribution and decay profile: one fracton emission
}

\author{
S. Alexander, O. Entin-Wohlman, R. Orbach
}

\section{To cite this version:}

S. Alexander, O. Entin-Wohlman, R. Orbach. Relaxation rate distribution and decay profile: one fracton emission. Journal de Physique Lettres, 1985, 46 (12), pp.549-554. 10.1051/jphyslet:019850046012054900 . jpa-00232558

\section{HAL Id: jpa-00232558 https://hal.science/jpa-00232558}

Submitted on 1 Jan 1985

HAL is a multi-disciplinary open access archive for the deposit and dissemination of scientific research documents, whether they are published or not. The documents may come from teaching and research institutions in France or abroad, or from public or private research centers.
L'archive ouverte pluridisciplinaire HAL, est destinée au dépôt et à la diffusion de documents scientifiques de niveau recherche, publiés ou non, émanant des établissements d'enseignement et de recherche français ou étrangers, des laboratoires publics ou privés. 
Classification

Physics Abstracts

$63.50-76.30-61.40$

\title{
Relaxation rate distribution and decay profile : one fracton emission
}

\author{
S. Alexander $\left({ }^{*}\right)$, O. Entin-Wohlman $\left({ }^{* *}\right)$ and R. Orbach \\ Department of Physics, University of California, Los Angeles, CA 90024, U.S.A.
}

(Reçu le 4 février 1985, accepté sous forme définitive le 23 avril 1985)

\begin{abstract}
Résumé. - Le taux de relaxation d'un état électronique localisé interagissant avec des états vibrationnels localisés (fractons, phonons localisés) est calculé pour le cas à un quantum de vibration. On examine en détail la statistique des états vibrationnels d'énergie et position appropriées qui peuvent participer à la relaxation d'un état électronique donné. On calcule la distribution de probabilité d'un quantum de taux de relaxation. La dépendance temporelle du profil de relaxation est plus lente qu'une décroissance exponentielle, mais plus rapide que toute décroissance en loi de puissance.
\end{abstract}

\begin{abstract}
The relaxation rate of a localized electronic state interacting with localized vibrational states (fractons, localized phonons) is calculated for the one-vibrational quantum case. Care is taken to deal with the statistics of finding the localized vibration of appropriate energy and position which can relax a given electronic state. The one quantum relaxation rate probability distribution is calculated. The time dependence of the relaxation profile is slower than exponential, but faster than any power law.
\end{abstract}

Relaxation of both localized [1] and extended [2] electronic states by running wave vibrational states (phonons) is well understood. The purpose of this Letter is to perform the same calculations for localized electronic states interacting with localized vibrational states. The electron localization we treat can arise from the inherent character of the electronic wave function (e.g. a localized magnetic impurity), or from the disorder of the underlying atomic structure. The latter results in electronic state localization, either by virtue of the geometrical properties of an atomically " pure » structure (e.g. a fractal network) or from imperfections which localize in the Anderson sense. The aspects of this paper which are new are the interaction of such electronic states with localized vibrational states. The latter can also be localized by virtue of the geometrical properties of the atomic network, in which case we refer to the vibrational excitations as fractons [3]. We shall implicitly consider only the vibrations of random fractal networks, for which localization of the vibrational states occurs if the fracton dimensionality, $\overline{\bar{d}}$, is less than 2 [4]. Ordered fractal networks (e.g. the Sierpinski gasket) intermix extended and localized vibrational states [5], greatly complicating the calculations. Further, they are not expected to be directly relevant to physical systems, while random fractal networks may [6]. Impurities in an otherwise transla-

(*) Permanent address : Racah Institute, The Hebrew University, Jerusalem, Israel.

(**) Permanent address : School of Physics and Astronomy, Tel Aviv University, Tel Aviv, Israel 69978. 
tionally invariant atomic network can also result in vibrational state localization [7]. We refer to these excitations as localized phonons. Because localized phonons are a special case of fractons, we shall first perform the calculation for fractons [3] and use the proper fractal indices ([3], [6] and [8]), in our derivation. This is both more general and also emphasizes our conviction that in the interesting experimental situations the relevant connected local geometry is best described by fractals.

As compared to calculations performed with extended vibrational states, the consequences of vibrational localization are profound. For the latter, the relaxation rate at different electronic sites is different because of fluctuations in the distribution of suitable (i.e., energy conserving) vibrational states near the electronic site. We shall show that this results in a very broad distribution of relaxation rates, and in a remarkable relaxation time profile : faster than any power law but slower than an exponential or stretched exponential.

We consider here the simplest of a multitude of possibilities : an excited localized electronic state relaxing at $T=0$ by the emission of a single fracton. This would correspond in conventional notation to the one phonon relaxation process; hence we shall designate it as the " one fracton » relaxation process. The case [7] of localized phonons will be carried out at the end of this Letter.

Fracton excitations are expected to describe the higher energy portion of the vibrational spectrum in a disordered system [6]. A one fracton relaxation process would be expected to influence electronic transitions at the same energy. A subsequent analysis by us [9] of the two fracton (Raman) relaxation case will be more relevant to spin-lattice relaxation $\left(T_{1}\right)$ in an amorphous material [10].

We believe [6] that vibrational excitations of cross-linked polymers, amorphous solids, and irradiated quartz exhibit a crossover from phonon to fracton character above a crossover frequency. As a consequence, we feel that relaxation of localized electronic states involving transitions of energy greater than this crossover energy should be described by the time dependence calculated in this Letter. Examples would be the time dependence of the relaxation of electron or hole excitations in amorphous semiconductors (known to be faster than power law [11]), and excited state relaxation in fluorescent line narrowing experiments on paramagnetic impurities in glasses.

As a consequence of localization of the vibrational state, the electronic relaxation rate must be described by a broad probability density, very similar to that obtained for variable range hopping in one-dimensional metals [12]. We shall assume exponential localization of the vibrational states. The statistical problem posed in finding the energy conserving lattice vibration is quite analogous to "pseudo-unimolecular » reactions in the absence of diffusion [13]. Indeed, the explicit time profile we shall obtain is identical with that of Inokuti and Hirayama [14] when we reduce our results to the case of localized phonons (Euclidean limit).

We calculate the probability density for the largest electronic relaxation rate experienced by a localized electronic state via the one fracton interaction. The electronic state emits a fracton of energy $\omega_{\alpha}$ and makes a transition to an energy state differing from the initial state by an energy $\omega_{0}$. Energy conservation will force $\omega_{\alpha}=\omega_{0}$. The time dependence of the electronic level occupation will then be the Laplace transform of the probability density.

We label each fracton state with a mode index $\alpha$, and take the form of the fracton wave function at energy $\omega_{\alpha}$ to be :

$$
\phi_{\alpha}\left(\omega_{\alpha}\right)=\left(l_{\omega_{\alpha}}\right)^{-\bar{d} / 2} \exp \left[-\frac{1}{2}\left(L / l_{\omega_{\alpha}}\right)^{d_{\min }}\right]
$$

where $l_{\omega_{\alpha}}$ is the localization length of the vibrational state. This form for the wave function on a fractal network has been proven recently by Levy and Souillard [15]. For the purposes of this paper, we shall assume that equation (1) is appropriate to the fracton regime of the vibrational 
states of a percolating network. The exact nature of the spatial extent of fracton states on a percolating network is unknown. However, it does appear that only a single length scale is appropriate to percolation, and we know the fracton states to be localized [4]. For these reasons, we pose equation (1) as an appropriate fracton wave function which we shall use in our subsequent calculations. Should this assumption prove not to be correct, our formal treatment remains valid, and one would only have to insert the correct fracton wave function in our subsequent expressions.

Taking $\omega_{\alpha}=\omega_{0}$, the relaxation rate of the electronic state due to a fracton centred a distance $L$ away is proportional to

$$
\begin{aligned}
& W\left(\omega_{0}, L\right) \propto \omega_{0}^{2 q-1}\left(l_{\omega_{0}}\right)^{-\bar{d}} \operatorname{coth}\left(\beta \omega_{0} / 2\right) \frac{1}{\delta_{L}} \exp \left[-\left(L / l_{\omega_{0}}\right)^{d_{\min }}\right] \equiv \\
& \equiv W_{\max }\left(\omega_{0}\right)[\delta(0) / \delta(L)] \exp \left[-\left(L / l_{\omega_{0}}\right)^{d_{\min }}\right],
\end{aligned}
$$

where $\delta(L)$ is the sum of the energy widths of the electronic and fracton states.

On a fractal, $\bar{d}$ is the fractal (Hausdorff) dimensionality, $d_{\min }$ an index which relates the Euclidean length $L$ to the " chemical length " $l$, with $d_{\min }$ defined in [16], and $l_{\omega_{\alpha}} \propto \omega_{\alpha}^{-\bar{d} / \bar{d}}$ is the localization length with $\overline{\bar{d}}$ the fracton dimension [3]. This relationship was posed initially by Alexander and Orbach [3], and arises from the solution to the diffusion problem on a fractal network of Gefen et al. [17] in combination with the mapping of the diffusion problem onto the vibrating network [18]. It is valid in the short length scale regime for random fractals, and thus in the fracton regime for vibrational states.

The index $q$ in equation (2) results from the spatial derivatives of $\phi_{\alpha}\left(\omega_{\alpha}\right)$ [1]. Usually [1] one has $q=1$, but on fractals it is usually more correct to put $q=(\overline{\bar{d}} / \bar{d}) d_{\min }(<1)$. We shall discuss these parameters elsewhere [9]. For our purposes here they can be regarded as parameters which make the argument more general without complicating the derivation significantly. It is now necessary to sum equation (2) over the allowed fracton levels. The probability that there is a fracton of energy $\omega_{0}$ between a distance $L$ and $(L+\Delta L)$ from the electronic site is proportional to $\Delta L \bar{d}(L)^{d-1} N\left(\omega_{0}\right) \delta(L)$, where $N\left(\omega_{0}\right)$ is the fracton energy density of states, and $\delta(L)$ is the level width introduced in (2). We wish to calculate the probability density for the largest relaxation rate at a particular electronic site. That means that we must calculate the probability that no other fracton state of energy $\omega_{0}$ is closer than $L$. This is given by the product

$$
\prod_{L^{\prime} \leqslant L}\left[1-\bar{d}\left(L^{\prime}\right)^{\bar{d}-1} \Delta L^{\prime} N\left(\omega_{0}\right) \delta\left(L^{\prime}\right)\right]=\exp \left[-\int_{0}^{L} \mathrm{~d} L^{\prime} \bar{d} N\left(\omega_{0}\right) \delta\left(L^{\prime}\right)\left(L^{\prime}\right)^{\bar{d}-1}\right]
$$

This is all that is required to calculate the probability density for the largest electronic relaxation rate experience at a given electronic site. However, the relaxation rate from equations (2) and (3) contains the level widths of both the electronic and fracton states in the factor $\delta(L)$. In order to carry out the integral in equation (3), it is necessary to specify the $L$ dependence of the level widths. For simplicity, we limit the discussion here to two cases : a) $\delta(L)$ is a constant $\delta$, independent of $L$, and b) $\delta(L)$ is just the self-consistent electronic relaxation rate $W$. The former would be relevant to, for example, a rapid lifetime broadening of the fracton states; the latter to no intrinsic fracton energy width. We shall outline the calculation of case a) here, and only state the results for case b) below.

For $\delta(L)=\delta$, independent of $L$, we find from equations (2) and (3) :

$$
\begin{array}{r}
P_{\mathrm{a}}\left(\omega_{0}, W\right) \mathrm{d} W=\left(\bar{d} / d_{\min }\right) c_{1}\left(\omega_{0}\right)\left[\ln \left(W_{\max } / W\right)\right]^{\left(\bar{d} / d_{\min }\right)-1} \times \\
\times \exp \left\{-c_{1}\left(\omega_{0}\right)\left[\ln \left(W_{\text {max }} / W\right)\right]^{\left(\bar{d} / d_{\text {min }}\right)}\right\} \mathrm{d} W / W,
\end{array}
$$


where $W$ ranges from 0 to $W_{\max }$ and $c_{1}\left(\omega_{0}\right)=N\left(\omega_{0}\right) \delta\left(l_{\omega_{0}}\right)^{\bar{d}}=\overline{\bar{d}} \delta / \omega_{0}$ (for fractons). The time dependence of the population of the initial electronic state is given by

$$
P_{\mathrm{a}}\left(\omega_{0}, t\right)=\int_{0}^{W_{\max }} \mathrm{d} W \exp (-W t) P_{\mathrm{a}}\left(\omega_{0}, W\right) .
$$

We introduce the dimensionless time $\tau=W_{\max } t$, and define $\tau_{1}=\left(d_{\min } / \bar{d}\right) \tau / c_{1}\left(\omega_{0}\right)$. Inserting equation (4) into equation (5), and carrying out the usual saddle point approximation for integrals of this kind, we find to leading order at long times,

$$
P_{\mathrm{a}}\left(\omega_{0}, \tau_{1}\right) \propto\left[2 \pi\left(\bar{d} / d_{\min }\right) c_{1}\left(\omega_{0}\right)\right]^{1 / 2}\left(\ln \tau_{1}\right)^{\left(\bar{d}-d_{\min }\right) / 2 d_{\min }} \tau_{1}^{-c_{1}\left(\omega_{0}\right)\left[\ln \tau_{1}\right]^{\left(\bar{d} / d_{\min }\right)-1},},
$$

neglecting logarithmic corrections of the order $\ln \left(\ln \tau_{1}\right)$ to the saddle point [9]. This remarkable result shows that the decay of the excited state population is slower than exponential, but more rapid than any power law.

Case b), where $\delta(L)$ is given self-consistently by $W\left(\omega_{0}, L\right)$, is considerably more complicated. The integral of the quantity analogous to equation (4) is less than unity, showing that some electronic states simply do not relax. Defining $c_{2}\left(\omega_{0}\right)=N\left(\omega_{0}\right) W_{\max }\left(l_{\omega_{0}}\right)^{\bar{d}}=\overline{\bar{d}} W_{\max } / \omega_{0}$ (for the fracton case), one finds after extensive algebra, using the saddle point method again,

$$
P_{b}\left(\omega_{0}, \tau\right) \propto\left\{\exp \left[-a c_{2}\left(\omega_{0}\right)\right]\right\}\left\{1+c_{2}\left(\omega_{0}\right)\left(2 \pi / e^{2}\right)^{1 / 2}\left(\bar{d} / d_{\min }\right)(\ln \tau)^{\left[\left(\bar{d} / d_{\min }\right)-1\right]} / \tau\right\},
$$

where $a$ is a number of order unity. The decay is close to a power law, with clear evidence for non-relaxing electronic states.

Finally, one can easily make the transition to localized phonons by setting [3] $\overline{\bar{d}}=\bar{d}=d$, and $d_{\min }=1$. Redefining the constants : $c_{1}^{\mathrm{ph}}\left(\omega_{0}\right)=N_{\mathrm{ph}}\left(\omega_{0}\right) \xi^{d} \delta, c_{2}^{\mathrm{ph}}\left(\omega_{0}\right)=N_{\mathrm{ph}}\left(\omega_{0}\right) \xi^{d} W_{\max }^{\mathrm{ph}}$, where $\xi$ is the phonon localization length, assumed independent of $\omega_{0}$ one can substitute immediately into equations (6) and (7) for cases a) and b), respectively. For example, for case a) in $d=3, P_{\mathrm{a}}(\tau) \propto \tau^{-(\ln \tau)^{2}}$.

Another interesting feature of the relaxation rate probability density is its average value. This will be important when the spin-spin interaction is in excess of $W_{\max }$. Under these conditions of strong cross relaxation, all spins will relax exponentially in time at an average relaxation rate which can be calculated directly from equation (2). We integrate this equation over all fracton modes to obtain,

$$
\langle W\rangle \sim N_{\mathrm{fr}}\left(\omega_{0}\right) \omega_{0}^{2 q-1} \operatorname{coth}\left(\beta \omega_{0} / 2\right),
$$

where we have used the fact that the wave function equation (1) is normalized. An entirely equivalent result can be obtained from the first moments of the probability densities equations (6) and (7). It will be shown elsewhere [9] that the results are equivalent to equation (8). The dependence of the average relaxation rate upon the magnitude of the energy transfer $\omega_{0}$ is,

$$
\begin{array}{ll}
\langle W\rangle \sim \omega_{0}^{2 q+\bar{d}-2}, & \beta \omega_{0} \gg 1, \\
\langle W\rangle \sim \omega_{0}^{2 q+\bar{d}-3} k_{\mathrm{B}} T, & \beta \omega_{0} \ll 1 .
\end{array}
$$

To make comparison with the usual results for (extended) phonon vibrational states [1], one need only replace $\overline{\bar{d}}$ in equations (8) and (9) by $d, q$ by 1 , and $N_{\mathrm{fr}}\left(\omega_{0}\right)$ by $N_{\mathrm{ph}}\left(\omega_{0}\right) \propto \omega_{0}^{d-1}$. One sees that the temperature dependence will remain the same, but the frequency dependence is rather different.

Our principal results (Eqs. (6) to (8)) have taken explicit account of fracton states at a distance $L$ from the electronic site to calculate the probability density for the largest relaxation rate. In 
principle, the contributions to $W\left(\omega_{0}, L\right)$ from fractons at distances greater than $L$ need consideration as well, lest their sum overwhelm our calculated result for the largest relaxation rate. Space limitations preclude presentation of the full analysis here [9]. For case a), when $c_{1}\left(\omega_{0}\right)=\overline{\bar{d}} \delta / \omega_{0}$ is small, only small logarithmic corrections are present in the re-scaling of $W$ and for the probability density $P_{\mathrm{a}}\left(\omega_{0}, \tau_{1}\right)$ of equation (6). We have forced a fracton to be at a distance $L$ from the electronic site in equation (2). The remaining fractons are at much larger distances statistically, and their contributions can be shown [9] to be small. For $c_{1}\left(\omega_{0}\right)$ large, the transition rate $W$ is strongly renormalized and the form of $P_{\mathrm{a}}\left(\omega_{0}, \tau_{1}\right)$ contains significant logarithmic corrections. Similar results are found for case b).

These results demonstrate that, contrary to the single electronic relaxation rate usually obtained for extended vibrational states, vibrational localization leads to a probability density for the electronic relaxation rate. We have calculated first the probability density for the relaxation rate, and then used it to calculate the relaxation time profile for the initially occupied electronic state. These calculations should be relevant to electron or hole excitation decay in amorphous semiconductors, and to optical transitions for localized electronic centres in glasses and polymers. While we have no direct evidence at this time to substantiate our relaxation model calculations, there is at least some evidence [11] that non-exponential time dependences are observed for excitations in amorphous semiconductors. The observed decay is faster than power law, in the same direction as predicted by equation (6). Indeed, we suggest that localization of vibrational states can be determined from the time dependence exhibited by the emission profile for an electronic transition in an amorphous or polymeric host.

The frequency (and temperature) dependence of the one fracton relaxation rate comes in through the $c_{\mathrm{i}}\left(\omega_{0}\right)$ and $W_{\text {max }}\left(\omega_{0}, T\right)$. We have considered the case where $\hbar \omega_{0} \gg k_{\mathrm{B}} T$ above, so that no explicit temperature dependence is exhibited in our results. The two fracton (Raman) process exhibits behaviour qualitatively similar to (6), with a much richer temperature dependence. Details will be given in [9].

\section{Acknowledgments.}

This research has been supported by the National Science Foundation through grant No. DMR 81-15542, DMR 84-12898, and INT 83-12985.

\section{References}

[1] Orbach, R., Stapleton, H. J., Electronic Paramagnetic Resonance, ed. by S. Geschwind (Plenum Press, New York) 1972, p. 121.

[2] Ziman, J., Electrons and Phonons (Oxford University Press, New York) 1962.

[3] Alexander, S. and Orbach, R., J. Physique Lett. 43 (1982) L-625 ;

Grest, G. and Webman, I., J. Physique Lett. 45 (1984) L-1155;

Rammal, R., J. Physique 45 (1984) 191.

[4] Rammal, R. and Toulouse, G., J. Physique Lett. 44 (1983) L-13.

[5] Domany, E., Alexander, S., Bensimon, D. and Kadanoff, L. P., Phys. Rev. B 28 (1983) 3110 ;

Rammal, R., Phys. Rev. B 28 (1983) 4871 ;

SOUTHERN, B. W. and DOUCHANT, A., in preparation (1985);

Douchant, A., M. Sc. Thesis, University of Manitoba, Winnipeg, Canada (1985).

[6] Alexander, S., Laermans, C., Orbach, R. and Rosenberg, H. M., Phys. Rev. B 28 (1983) 4615.

[7] John, S., Sompolinsky, H. and Stephen, M. J., Phys. Rev. B 27 (1983) 5592.

[8] Alexander, S., Ann. Israel Phys. Soc. 5 (1983) 144.

[9] AleXander, S., Entin-Wohlman, O. and Orbach, R., J. Physique Lett. 46 (1985) L-555. 
[10] Stapleton, H. J., Allen, J. P., Flynn, C. P., Stinson, D. G. and Kurtz, S. R., Phys. Rev. Lett. 45 (1980) 1456 ;

Allen, J. P., Colvin, J. T., Stinson, D. G., Flynn, C. P. and Stapleton, H. J., Biophys. J. 38 (1982) 299.

[11] TAUC, J., private communication.

[12] Alexander, S., Phys. Rev. B 26 (1982) 2956.

[13] Blumen, A., Zumofen, G. and Klafter, J., Structure and Dynamics of Molecular Systems, ed. by R. Daudel (Reidel Publishing Co., Dordrecht, The Netherlands), to appear in January, 1985.

[14] Inokuti, M. and Hirayama, F., J. Chem. Phys. 43 (1965) 1978.

[15] LEVY, Y. and Souillard, B., unpublished.

[16] Middlemiss, K. M., Whittington, S. G. and Gaunt, D. S., J. Phys. A 13 (1980) 1835 ;

Pike, R. and Stanley, H. E., J. Phys. A 14 (1981) L169;

Hong, D. C. and Stanley, H. E., J. Phys. A 16 (1983) L475;

Hong, D. C. and Stanley, H. E., J. Phys. A 16 (1983) L525;

Havlin, S. and Nossal, R., J. Phys. A 17 (1984) L427;

Vannimenus, J., Nodal, J. P. and Martin, C., J. Phys. A 17 (1984) L351;

Havlin, S., Djordjevic, Z. V., Majid, I., Stanley, H. E. and Weiss, G. H., Phys. Rev. Lett. 53 (1984) 178 ;

Hermann, H. J., Hong, D. C. and Stanley, H. E., J. Phys. $A 17$ (1984) L261 ;

Rammal, R., Angles D'Auriac, J. C. and Benoit, A., J. Phys. A 17 (1984) L491.

[17] Gefen, Y., Aharony, A. and Alexander, S., Phys. Rev. Lett. 50 (1983) 77.

[18] Alexander, S., Bernasconi, J., Schneider, W. and Orbach, R., Rev. Mod. Phys. 53 (1981) 175. 